injection, and were less sensitive to morphine in both pain-tests $(\mathrm{MED}=6.0 \mathrm{mg} / \mathrm{kg})$. LEW rats were completely insensitive to morphine in the hot plate, in contrast to reversal of CFA-induced hyperalgesia $(M E D=3.0 \mathrm{mg} / \mathrm{kg}$ ). Additionally, neuropathic sensitivity developed with a later onset and less robustly in this strain after SNI. All strains tested in LMA exhibited sedative effects at $3.0 \mathrm{mg} / \mathrm{kg}$.

Conclusions: Sensory phenotyping in response to acute, inflammatory and neuropathic pain, and sensitivity to morphine in these strains indicates that different pathophysiological mechanisms are engaged after injury. This could have profound implications for translating preclinical drug discovery efforts into pain-patients.

http://dx.doi.org/10.1016/j.sjpain.2017.04.063

\section{Choice of rat strain in pre-clinical pain-research - Does it make a difference for translation from animal model to human condition?}

S. Hestehave ${ }^{\mathrm{a}, \mathrm{b}, *}$, G. Munro ${ }^{\mathrm{a}, \mathrm{c}}, \mathrm{T}$. Brønnum-Pedersen $^{\mathrm{d}}$, A.M. Heegaard ${ }^{\mathrm{e}}$, K.S.P. Abelson $^{\mathrm{b}}$

a Department of In Vivo Neurodegeneration, $H$. Lundbeck A/S, Valby, Denmark

${ }^{\mathrm{b}}$ Department of Experimental Medicine, Faculty of Health and Medical Sciences, University of Copenhagen, Denmark

${ }^{\mathrm{c}}$ Department of Neurology, Danish Headache Center, Glostrup Research Institute, Glostrup, Denmark

${ }^{d}$ Department of Non-Clinical Safety Research, $H$. Lundbeck A/S, Valby, Denmark

e Department Drug Design and Pharmacology, Faculty of Health and Medical Sciences, Copenhagen,

Denmark

E-mail address: sara.kristensen@sund.ku.dk(S. Hestehave).

Aims: Translating preclinical drug-efficacy of analgesics from animal models to humans has proven challenging with many failures. Reasons are likely multifaceted, but lack of sufficiently rigorous study design, and phenotypical relevant animal models may be part of the explanation. Chronic pain is often associated with substantial comorbid burden, consisting of changes in affective state and cognitive impairment amongst other behavioral disturbances. Accordingly, many preclinical pain research activities have started to include assessment of comorbidity as a possible experimental outcome measure, but surprisingly, little consideration has been paid to the influence of animal-related factors to pain models. To address this essential issue, we have embarked on several comparative experiments in different pain-models, comparing Sprague Dawley's (SD) from two different vendors with different inbred rat strains (Lewis (LEW), Fisher (F344) and Wistar Kyoto (WKY)) selected based on reported stress, depression, inflammatory and pain phenotypes.

Methods: Male rats were characterized in acute (hot-plate), inflammatory (Complete Freund's Adjuvant(CFA)) and neuropathic (Spared Nerve Injury (SNI)) pain models, with dose-response to morphine $(0.3-6.0 \mathrm{mg} / \mathrm{kg})$ in hot-plate, CFA-induced hyperalgesia, and a locomotor motility-assay (LMA).

Results: F344 and SD's were sensitive to morphine in hotplate and CFA (Minimum Effective Dose (MED) $=3.0 \mathrm{mg} / \mathrm{kg}$ ). WKY rats developed a less robust mechanical hypersensitivity after CFA 